\title{
Pesquisa e produção bibliográfica em terapia ocupacional: contribuições ao debate sobre parâmetros de avaliação da produção acadêmica brasileira*
}

\author{
Research and bibliographic production on \\ occupational therapy: contributions to the debate \\ on parameters for the evaluation of brazilian \\ academic production
}

Fátima Corrêa Oliver ${ }^{1}$

OLIVER, F. C. Pesquisa e produção bibliográfica em terapia ocupacional: contribuições ao debate sobre parâmetros de avaliação da produção acadêmica brasileira Rev. Ter. Ocup. Univ. São Paulo, v. 19, n. 2, p. 108-120, maio/ago. 2008.

\begin{abstract}
RESUMO: O estudo caracterizou produção bibliográfica (2001/2007) de grupos de pesquisa, coordenados por terapeutas ocupacionais, no Diretório do CNPq. Foram identificados 17 grupos, 12 em instituições públicas. Houve ampliação (68\%) de participantes dos grupos (de 2000 a 2007). Os grupos contam com 48 doutores; 12 vinculados a programas de pós-graduação estrito senso. Os pesquisadores publicaram 21 livros, 159 capítulos de livros e 348 artigos. Percebeu-se maior número de artigos publicados em periódicos nacionais sendo $146(41,7 \%)$ em periódicos classificados como Nacional B ( 97 - 29,8\% na Revista de Terapia ocupacional da USP), 16 (4,6\%) como Nacional A. Os artigos em periódicos de circulação internacional representaram $21,7 \%$, sendo 54(15,5\%) Internacional C, $11(3,1 \%)$ Internacional B e $11(3,1 \%)$ como Internacional A. Os pesquisadores publicaram $153(57,7 \%)$ dos 265 artigos dos periódicos de terapia ocupacional, importante contribuição também para sustentação desses veículos. Houve dispersão de artigos em grande número de periódicos nacionais, o que divulga a área, mas pode dificultar a consolidação de periódicos de terapia ocupacional. As mudanças positivas auxiliam avaliação da contribuição de autores brasileiros da área e assim melhor qualificar a produção bibliográfica e a pesquisa em terapia ocupacional.
\end{abstract}

DESCRITORES: Terapia ocupacional. Pesquisa. Bibliografia. Grupos de pesquisa. Indicadores de produção científica.

\footnotetext{
* Parte das discussões deste artigo foram apresentadas durante o XI Encontro Nacional de Docentes de Terapia Ocupacional realizado em São Paulo de 10 a 12 de setembro de 2008

1 Doutora em Saúde Pública, docente do Depto. de Fisioterapia Fonoaudiologia e Terapia Ocupacional da Faculdade de Medicina da Universidade de São Paulo

Endereço para correspondência: Rua Cipotânea, 51 Cidade Universitária CEP 05360-160 São Paulo, SP. E-mail: fcoliver@usp.br
} 


\section{INTRODUÇÃO}

$\mathrm{O}$ estudo pretende contribuir para o debate sobre a pesquisa e a qualificação e desenvolvimento da produção bibliográfica em terapia ocupacional no Brasil ampliando as discussões trazidas por estudo anteriormente realizado (BARROS; OLIVER, 2003).

A terapia ocupacional é um campo profissional que tem se consolidado desde a regulamentação da profissão em 1969, e mais intensamente, a partir dos anos 1980, com a institucionalização do currículo mínimo para a graduação e, nos anos 2000, com as diretrizes curriculares. Profissionais da área têm tido papel importante no delineamento tanto do currículo mínimo como das diretrizes curriculares através do movimento docente e das associações profissionais. Nos fóruns coletivos, como os Encontros Nacionais de Docentes e os Congressos Brasileiros de Terapia Ocupacional, esses temas têm sido pautados e discutidos apoiando a ação de representantes junto ao Ministério da Educação. Assim, o esforço de docentes de terapia ocupacional para propor e acompanhar a implantação de currículos e dos processos de avaliação do ensino superior tem sido permanente, para procurar garantir a implantação de ensino de qualidade no país.

O crescimento do número de cursos de graduação, principalmente a partir de 1998, recoloca desafios para docentes e profissionais no que diz respeito à estruturação e consolidação do ensino em diferentes regiões do país, e nesse sentido a questão da capacitação docente e do desenvolvimento de ensino articulado à pesquisa e extensão de serviços à comunidade têm importância fundamental (EMMEL, 2004).

Em 2004 havia, no país, 39 cursos, 9 deles em universidades públicas (TIRADO et al., 2004 p. 497), e em julho de 2008, dados do Instituto Nacional de Estudos e Pesquisas Educacionais Anísio Teixeira (INEP, 2008) identificavam 63 cursos de graduação, a maior parte nos estados de São Paulo e Minas Gerais. Nessa data, 12 cursos se desenvolviam em universidades públicas, instituições onde têm sido mais favoráveis as condições para o desenvolvimento de pesquisa articulada ao ensino de graduação e à extensão de serviços à comunidade.

Um campo profissional pode se consolidar à medida que discute seus princípios e fundamentos históricos, teóricos e metodológicos, que reflete sobre sua inserção institucional e política no âmbito do desenvolvimento das políticas assistenciais, ou seja à medida que pesquisa e produz conhecimento. O campo da terapia ocupacional conta com poucos atores, em setembro de 2008 havia no país 11.228 profissionais habilitados e credenciados junto ao sistema de conselhos regionais e federal (COFFITO, 2008) e em 2004, cerca de 460 docentes (EMMEL, 2004)

Estudar a produção bibliográfica na área é uma das maneiras possíveis de se conhecer as atividades de pesquisa realizadas por terapeutas ocupacionais, que se constituíram como parte do processo de capacitação docente, principalmente, para aqueles vinculados às universidades públicas (EMMEL; LANCMAN, 1998, a,b; MAGALHÃES, 1998). São da década de 1990, o desenvolvimento de parte dos estudos de mestrado e de doutorado realizados em programas de pós-graduação no Brasil, nas áreas de educação, saúde coletiva, ciências sociais, psicologia, comunicações e artes e, em menor número, fora do país em terapia ocupacional ou áreas afins. A opção pela capacitação docente no país fundava-se na perspectiva de diálogo com a produção do conhecimento sobre os principais problemas e demandas populacionais a serem enfrentados pelos terapeutas ocupacionais brasileiros. Assim, a partir da capacitação de docentes em diferentes campos do conhecimento seria possível conhecer, analisar e enfrentar os desafios colocados para a área no contexto nacional (BARROS; OLIVER, 2003).

A participação da autora nesse processo, sem a pretensão de discutir ou analisar sistematicamente o extenso campo de preocupações de pesquisa dos docentes, pode observar algumas tendências de estudos tais como: a constituição histórica e metodológica da terapia ocupacional; as práticas de ensino e o currículo da graduação; as políticas públicas (saúde, educação e assistência social) em que se integravam atividades e programas de atenção; as perspectivas do sujeito com deficiências ou incapacidades, de familiares e/ou profissionais diante da deficiência, dos processos de tratamento e de reabilitação. Também são campo de preocupações de docentes e profissionais estudos sobre metodologia de intervenção empregada e desenvolvida para acompanhamento da infância, da adolescência, de jovens, adultos e idosos e suas interfaces na saúde, na educação, na reabilitação, incluindo diferentes modelos de atuação em terapia ocupacional. Por outro lado, também, tem existido preocupação de docentes e profissionais com o estudo, planejamento e desenvolvimento de ajudas técnicas, de tecnologias de processos de atenção e de desenvolvimento de produtos (instrumentos de avaliação, equipamentos e adaptações).

Apesar das atividades de pesquisa estarem mais restritas aos profissionais vinculados à docência e do pequeno número de profissionais graduados em terapia ocupacional, percebe-se a ampliação e repercussão das preocupações com a formação para a pesquisa desde a graduação. Em algumas instituições de ensino superior, a pesquisa envolve estudantes em projetos de iniciação científica, apoiados pelas 
próprias instituições e/ou agências de fomento, como CNPq ou fundações estaduais de apoio à pesquisa. .

No entanto, esse percurso ainda não é suficiente para a construção de iniciativas de pesquisa mais sólidas com reflexos na formação de pesquisadores, que no caso brasileiro se dá, quase que exclusivamente, nos programas de pós-graduação, que têm sido implementados nas últimas décadas.

A partir de 1998, os parâmetros de avaliação da pósgraduação brasileira têm sido discutidos em suas diferentes dimensões (objetivos dos programas; tempo de formação de mestres e doutores; qualificação da produção bibliográfica, intercâmbio internacional entre outros). A avaliação da pósgraduação está associada à qualificação e à classificação dos programas com objetivo de concessão de recursos (bolsas, verbas de bancada entre outros). É nesse momento de reorganização da avaliação da pós-graduação que têm início as propostas de criação de programas de pós-graduação com participação de docentes de terapia ocupacional.

Assim, os parâmetros para criação de pós-graduação nas diferentes áreas do conhecimento: como constituição de linha de pesquisa, existência de doutores qualificados e demanda de capacitação docente, que predominavam até os anos 1990, passam a ser redimensionados com privilégio à pesquisa de base biomédica, prioridade na realização de mestrados e doutorados em 24 e 48 meses, respectivamente, bem como à divulgação de conhecimento a partir de artigos em periódicos com circulação internacional.

Por outro lado, o fato da terapia ocupacional compor a grande área de conhecimento da saúde, com tradição já estabelecida de programas de pesquisa e de pós-graduação com enfoque biomédico, tem dificultado a proposição e criação de programas específicos. A vinculação à grande área da saúde tem dificultado o desenvolvimento de estudos socioculturais para o enfrentamento dos problemas de pessoas ou grupos sociais acompanhados em terapia ocupacional que podem ser melhor conhecidos a partir da interface de pesquisa com as ciências humanas (psicologia, antropologia, sociologia,educação, comunicação e artes). Esses problemas, que têm se traduzido em estudos na área, não podem ser dimensionados apenas a partir do privilégio ao conhecimento biomédico e de suas estratégias de pesquisa e de qualificação da produção acadêmica e bibliográfica.

Os terapeutas ocupacionais têm contribuído para discutir e pesquisar alternativas para lidar com dificuldades e impossibilidades de construção de autonomia pessoal e social, de participação e desenvolvimento de atividades significativas para a constituição de sujeitos ativos, para facilitar processos de inclusão social lidando com a construção de vida cotidiana. Essa dimensão deve estar representada na pesquisa e na divulgação da produção bibliográfica promovendo o diálogo principalmente entre profissionais e pesquisadores do país e para isso também necessita de apoio financeiro das agências de fomento.

Este estudo pretende contribuir para a criação de estratégias de enfrentamento dessa realidade, conhecendo os percursos desenvolvidos por pesquisadores para divulgação científica e assim dar visibilidade à constituição da pesquisa em terapia ocupacional. Compreende-se a produção bibliográfica como um dos resultados das atividades de pesquisa, que possibilita o diálogo de um campo de conhecimento tanto internamente como na sua relação com a comunidade acadêmica e científica.

\section{Procedimentos metodológicos}

O estudo foi realizado a partir da identificação e caracterização da produção bibliográfica de pesquisadores participantes de grupos de pesquisa do diretório de grupos de pesquisa do Conselho Nacional de Desenvolvimento Científico e Tecnológico (CNPq). Foram escolhidos os grupos de pesquisa coordenados por terapeutas ocupacionais, vinculados a cursos de graduação, pois se compreendeu que nesses grupos o desafio da pesquisa está em aliar seu desenvolvimento à implementação do ensino, na maior parte das instituições, ensino de graduação.

Em novembro e dezembro de 2007, foram levantados os grupos de pesquisa no diretório de grupos de pesquisa do $\mathrm{CNPq}$, certificados pelas instituições de ensino superior. A partir da consulta aos currículos Lattes dos pesquisadores, em dezembro de 2007 e em junho de 2008, foi organizado banco de dados da produção bibliográfica realizada entre 2001 e 2007, que incluiu livros, capítulos de livros e artigos publicados em periódicos nesse período.

Os dados foram organizados em gráficos e planilhas para identificar participantes dos grupos de pesquisa, livros e capítulos de livros publicados, bem como artigos, segundo periódicos de divulgação e período. Os dados foram discutidos tendo em vista os processos de avaliação da produção bibliográfica e acadêmica desenvolvidos pela Capes e sua repercussão para a pesquisa e capacitação docente em terapia ocupacional.

Os grupos de pesquisa em terapia ocupacional: perfil de participantes e da produção bibliográfica

Em 2007, havia no Diretório de grupos de pesquisa do $\mathrm{CNPq}, 17$ grupos coordenados por terapeutas ocupacionais, distribuídos em 6 instituições de ensino superior sendo 12 grupos em instituições públicas, conforme apresentado no 
Quadro 1. Entre 2000 e 2007, permaneceram praticamente o mesmo número de instituições de ensino superior do período de 1985 - 2000. As Universidades Federais de São Carlos e de Minas Gerais mantiveram o mesmo número de grupos de pesquisa e na Universidade de São Paulo houve diminuição de sete para cinco grupos, com a transferência de um deles para o campus de Ribeirão Preto e a extinção de outro. No âmbito das instituições particulares houve a criação de mais um grupo de pesquisa na Pontifícia Universidade Católica de Campinas e também a extinção de grupos de duas instituições de ensino superior privadas, Universidade de Sorocaba e Universidade Potiguar. Em 2007, houve a inclusão de uma nova instituição, Fundação Bahiana para o Desenvolvimento das Ciências, com dois grupos de pesquisa.

A experiência acadêmica e de pesquisa dos docentes das diferentes instituições orientaram a criação e perfil de atividades desses grupos, alguns deles em atividade desde os anos 1990. Os 17 grupos atuais trabalham sobre temas e preocupações em diferentes campos, com multiplicidade de interesses e de atividades, melhor detalhadas nas suas linhas de pesquisa, que poderiam ser objeto de outros estudos.

O perfil dos participantes dos grupos de pesquisa se modificou no período de 2000 a 2007, conforme apresentado no Gráfico 1. Em cada ano, foi contabilizado o conjunto de participantes, ajustando-se os dados, pois havia pesquisadores que participavam em mais de um grupo de pesquisa, geralmente em instituições de ensino diferentes, o que sugere integração interinstitucional nas atividades de pesquisa.

No Gráfico 1, nota-se a diminuição do número total de participantes entre 2000 e 2002 e aumento de 2002 a 2007. Tendência semelhante foi observada entre os participantes graduandos/graduados/especialistas, mestrandos e doutorandos. Já para os doutores percebe-se o aumento maior entre 2000 e 2002 e menor entre 2002 e 2007, o que sugere a capacitação dos participantes dos grupos ao longo do período.

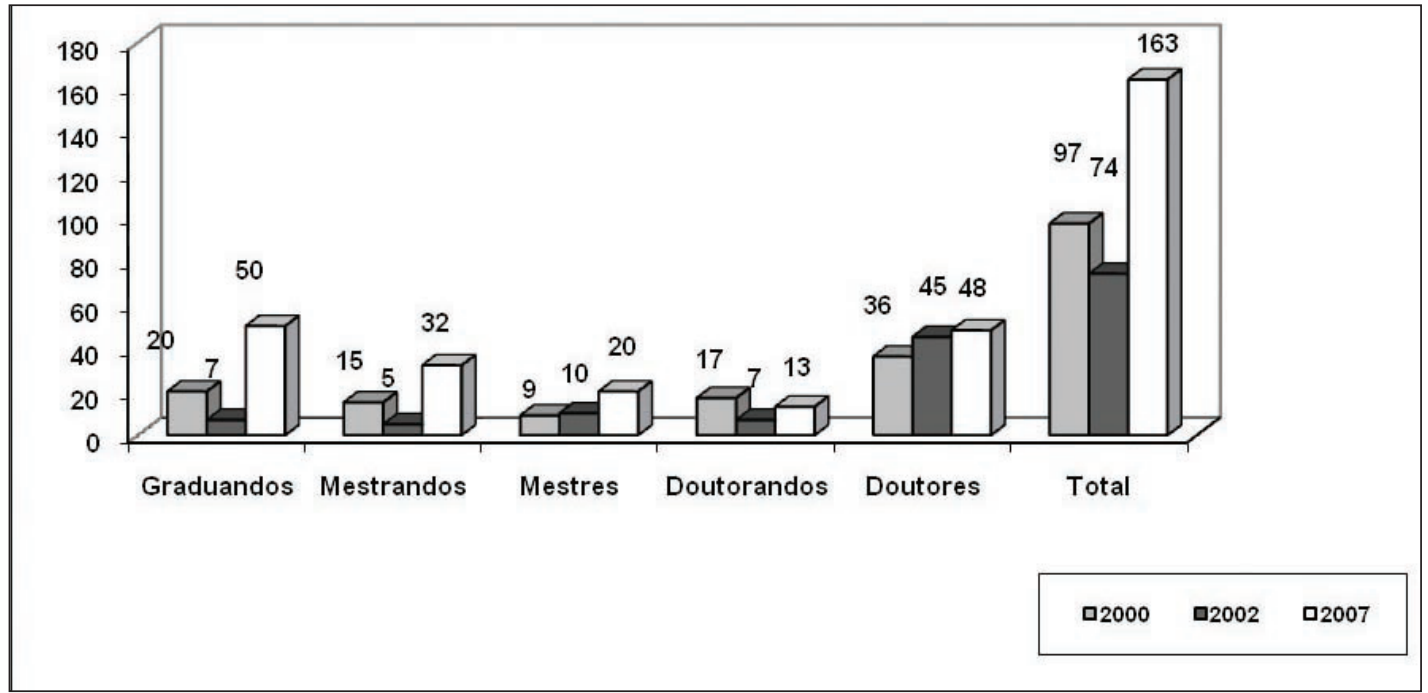

Gráfico 1 - Perfil dos participantes dos grupos de pesquisa no diretório de pesquisa do CNPq, segundo registros nos anos 2000, 2002, 2007

$\mathrm{Na}$ constituição dos grupos de pesquisa chama a atenção o aumento de cerca de seis vezes do número de graduandos/graduados/especialistas e de mestrandos entre 2002 e 2007, e a duplicação de mestres e doutorandos, o que pode sugerir a consolidação desses grupos envolvendo participantes com diferentes níveis de capacitação.

Entre os pesquisadores há 12 deles vinculados a programas de pós-graduação estrito senso. Nos programas de Ciências da Reabilitação na Universidade de São Paulo há 5 orientadores de mestrado e na Universidade Federal de
Minas Gerais há 2 orientadores de mestrado/doutorado. Já na Universidade Federal de São Carlos há 2 orientadores de mestrado e 2 orientadores de mestrado/doutorado no programa de Educação Especial e um orientador de mestrado/doutorado no programa de Fundamentos da Educação. Nesse período, os pesquisadores deram continuidade a sua capacitação sendo que nove deles desenvolveram estudos de pós-doutorado no Brasil ou em outros países (França, Canadá e Estados Unidos).

Neste estudo, ampliou-se o levantamento da produção 
bibliográfica em relação a estudo anterior (BARROS; OLIVER, 2003) com a inclusão de livros e capítulos de livros, além de artigos, conforme os dados apresentados no Gráfico 2. Assim, ficou evidente a duplicação do número de artigos publicados no período de 2001 a 2007 em relação ao período de 1985 a 2000 (BARROS; OLIVER, 2003, p. 56). Isso pode ser reflexo de maior atividade de pesquisa e sua divulgação nos grupos envolvendo participantes em diferentes momentos da formação acadêmica, já que o número de doutores foi quase constante no período.
Entre 2001 e 2007, foram publicados 21 livros, numa média de 3 livros/ano, o que sugere preocupação dos pesquisadores com a organização e produção de obras, que tenham um caráter mais de formação geral, que de divulgação de estudos mais pontuais, como é o caso de artigos. A produção de livros também possibilita apresentar o campo profissional e de intervenções em diálogo com profissionais do âmbito assistencial e com a formação, principalmente, em nível de graduação.

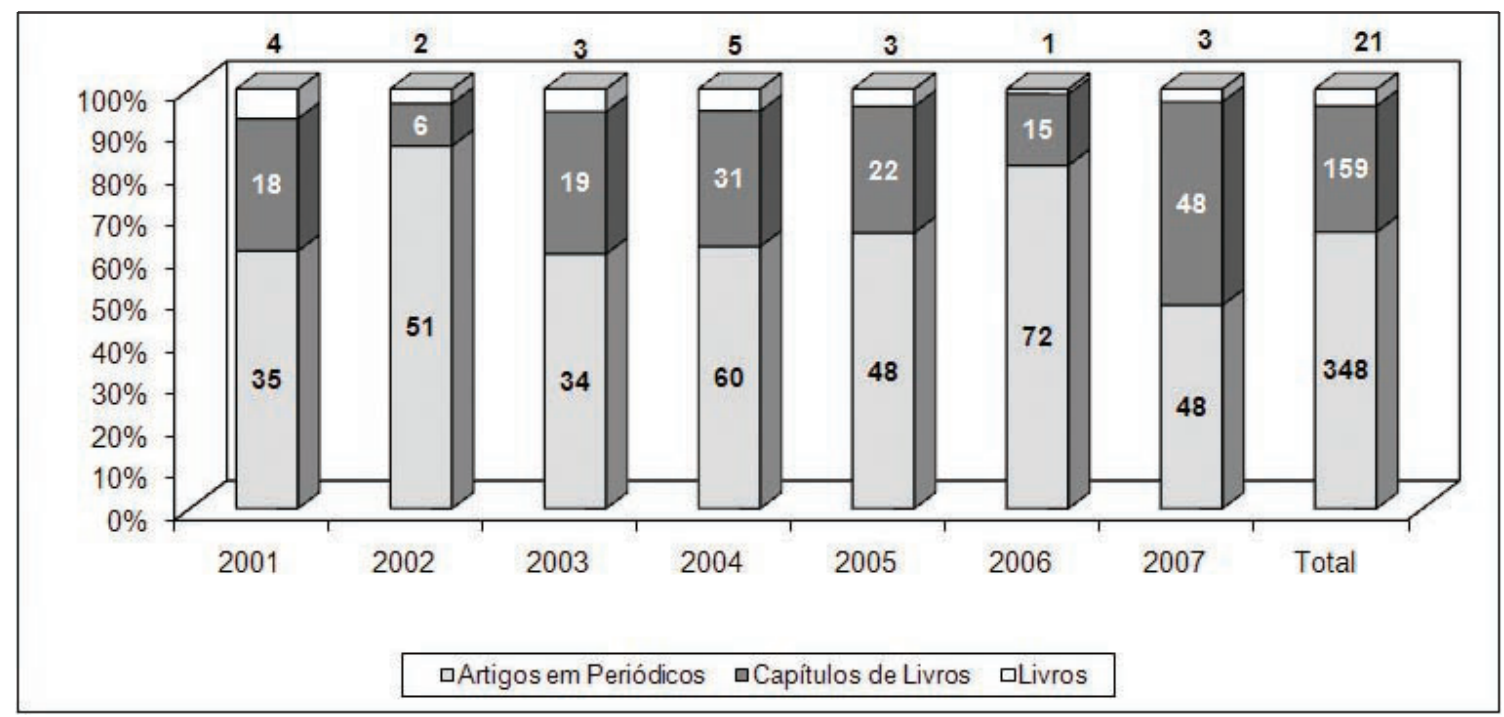

Gráfico 2 - Produção bibliográfica, segundo tipo e ano de publicação

Observou-se que, em 2002, o número de capítulos de livros foi cerca de oito vezes menor que o de artigos e, em 2006, cerca de cinco vezes menor. Já em 2001, 2003, 2004 e 2005, o número de capítulos de livros correspondeu a cerca da metade do número de artigos publicados, e em 2007 foi publicado o mesmo número de capítulos e artigos.

Ou seja, há preocupação e responsabilidade dos pesquisadores em lidar com a produção bibliográfica em suas diferentes dimensões, pois as necessidades de formação profissional e de implementação do campo de conhecimento não podem ser dimensionadas apenas pelas estratégias de avaliação dos organismos de fomento e de avaliação da pesquisa, que têm priorizado a produção bibliográfica na forma de artigos. Assim, os doutores e demais participantes dos grupos têm investido na constituição do campo, muitas vezes, em detrimento de sua habilitação individual para as atividades de pesquisa no âmbito do sistema de pós-graduação.

\section{A avaliação da produção intelectual no país e a produção bibliográfica em Terapia Ocupacional}

No Brasil, a Coordenação de Aperfeiçoamento de Pessoal de Nível Superior (CAPES) é responsável pelo credencimento e avaliação dos programas de pós-graduação estrito senso. Nesse âmbito, para a avaliação da produção intelectual foi criado um sistema de classificação denominado Qualis CAPES, discutido periodicamente entre representantes de diferentes áreas do conhecimento e de comitês de avaliação nomeados pela instituição.

Nesse cenário, a terapia ocupacional é parte da área 21 da CAPES juntamente com as áreas de Educação Física, Fisioterapia e Fonoaudiologia e tem restrita participação nas discussões sobre o sistema de avaliação.

Somente em 2007, representante da Rede Nacional de Ensino em Terapia ocupacional (RENETO) pode participar de discussões no Fórum de Coordenadores de Pós-graduação 
da área 21, sem contudo integrar comissões de discussão do sistema de classificação da produção intelectual.

Nesse sistema os periódicos são classificados em Qualis Internacional A, B e C e Nacional A, B e C e no momento deste estudo estava em vigor o Qualis 2004 -2006 (CAPES, 2008) que vem sendo discutido desde 2007.

No Qualis Internacional A e B estão periódicos nacionais ou estrangeiros que participem das bases do Institute for Scientific Information (ISI) dos Estados Unidos: Science Citation Index, Social Science Citation Index e Arts \& Humanities Citation Index reunidas na Web of Science (MUGNAINI et al., 2004, p.125) e que tenham contabilizado fator de impacto pelo Journal Citation Report (CAPES, 2008). O fator de impacto de um periódico é calculado pelo número de citações de artigos da revista realizados na própria revista em determinado período. A classificação em Qualis Internacional A e B na grande área da saúde se faz pela "mediana do índice de impacto do Journal Citation Report (JCR) dos periódicos específicos de cada área de avaliação" ... "aqueles com fator de impacto igual ou superior a mediana serão A e abaixo da mediana B" (CAPES, 2008).

$\mathrm{Na}$ área 21, até dezembro de 2007, foram considerados Internacional $\mathrm{A}$, aqueles periódicos com fator de impacto maior que 0,8 e Internacional $\mathrm{B}$ aqueles com fator de impacto menor que 0,8 . Nessa mesma área, os periódicos Internacional $\mathrm{C}$ devem apresentar: regularidade e periodicidade, padrão internacional quanto ao formato, qualidade de conteúdo, composição do corpo editorial e consultores, com distribuição de autorias e rigor na seleção de artigos e estarem catalogados em uma das bases: Medline, International Pharmaceutical Abstracts (IPA), International Nursing
Index (INI), Cumulative Index to Nursing \& Allied Health Literature (CINAHL), SportDiscus, ERIC, Tropical Diseases Bulletin, Sociological Abstracts e Planning/Policy \& Development (CAPES, 2008)

Tem sido considerado periódicos Nacionais A aqueles pertencentes à base de dados Scientific Electronic Library Online (SciELO). Os periódicos Nacionais B são aqueles catalogados na base Literatura Latino-americana e do Caribe de Ciências da Saúde (Lilacs); na Literatura em Ciências da Saúde publicada na Europa e Estados Unidos e outros países (Embase), na base de literatura de Psicologia (PsychLit), ou ainda aqueles editados por sociedades científicas representativas da área de avaliação. Os periódicos Nacionais C são aqueles que não atendem aos critérios anteriores,

A publicação de artigos em periódicos tem sido importante indicador de avaliação de atividades de pesquisa utilizado tanto pelas agências de fomento à pesquisa $(\mathrm{CNPq}$ e fundações estaduais de apoio) como para a criação de programas de pós-graduação estrito senso no país.

Nesse sentido, será descrita, a seguir, a produção bibliográfica em terapia ocupacional, compreendida como um dos resultados observados no delineamento de atividades de pesquisa e do desenvolvimento do ensino de graduação e, de forma ainda inicial, de pós-graduação em algumas instituições de ensino superior dos estados de São Paulo e Minas Gerais representadas pelos grupos de pesquisa estudados.

Entre 2001 e 2007, observou-se crescimento de 105\% do número de artigos publicados tanto em periódicos estrangeiros como em nacionais, pois entre 1985 e 2000 foram publicados 164 artigos e no período deste estudo 348 (BARROS; OLIVER, 2003) (Gráfico 3).

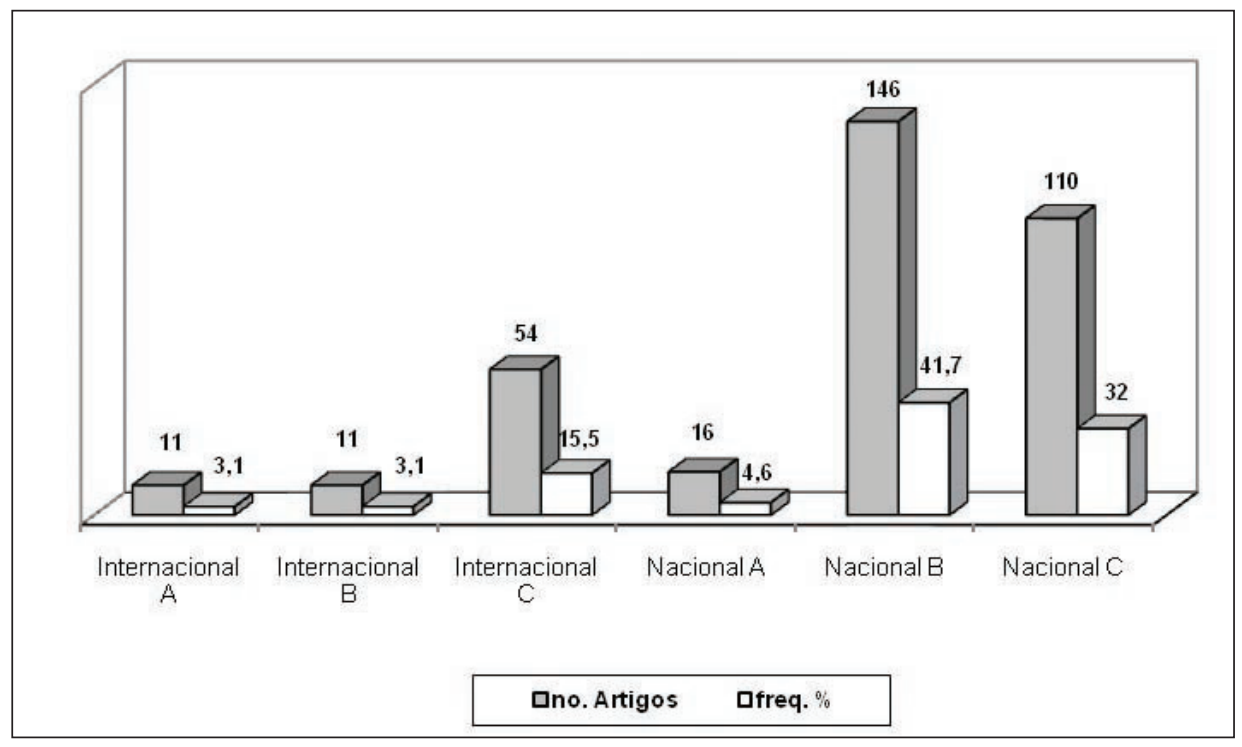

Gráfico 3 - Artigos publicados segundo qualificação da Capes, 2001 a 2007 
OLIVER, F. C. Pesquisa e produção bibliográfica em terapia. Rev. Ter. Ocup. Univ. São Paulo, v. 19, n. 2, p. 108-120, maio/ago. 2008.

Os participantes dos grupos de pesquisa publicaram 23 artigos em periódicos estrangeiros, resultado semelhante aos 24 artigos publicados entre 1985 e 2000. Ao mesmo tempo, percebeu-se aumento de $124 \%$ do número de artigos divulgados em periódicos nacionais (de 145 entre 1985 e 2000 para 325 entre 2001 e 2007), o que tem ampliado a divulgação de pesquisas, privilegiando o diálogo com veículos nacionais, como seria de se esperar também para uma área em constituição.

$\mathrm{O}$ crescimento do número de artigos deveu-se ao aumento daqueles publicados em periódicos nacionais, indexados pelas bases de dados Lilacs e SciELO, conforme Tabela 1. Esses artigos estão distribuídos em 23 periódicos, e concentrados na Revista de Terapia Ocupacional da USP, o que confirma a tendência do período anterior e reafirma a importância desse periódico como veículo de divulgação científica da área. Como entre 1985 e 2000, observou-se artigos publicados em diferentes campos do conhecimento como educação, psicologia, além daqueles em saúde (BARROS; OLIVER, 2003).

Tabela 1 - Artigos publicados em periódicos nacionais, classificados pela Capes como Qualis A e B Nacional, segundo indexadores, 2001 a 2007

\begin{tabular}{|c|c|c|c|}
\hline Periódicos Nacionais & No. Artigos & Indexadores & Qualis Capes \\
\hline 1. Revista de Terapia Ocupacional da USP/SP & 97 & Lilacs, Latindex & Nacional B \\
\hline 2. Temas sobre Desenvolvimento & 21 & Lilacs & Nacional B \\
\hline 3. O mundo da Saúde & 10 & Lilacs & Nacional B \\
\hline 4. Imaginário & 3 & Lilacs & Nacional B \\
\hline 5. Saúde Em Revista & 2 & Lilacs, Sportdiscus, Latindex & Nacional B \\
\hline 6. Acta Fisiátrica & 2 & Lilacs & Nacional B \\
\hline 7. Boletim de Psicologia & 2 & Lilacs, Index Psi; PePsic & Nacional B \\
\hline 8. Psychê & 1 & Lilacs, Index Psi, Clase, Psicodoc, PePsic & Nacional B \\
\hline 9. Reabilitar & 1 & Lilacs & Nacional B \\
\hline 10. Jornal Brasileiro de Endo/Perio & 1 & LILACS Latindex & Nacional B \\
\hline 11. Revista Brasileira de Biomecânica & 1 & Lilacs, SportDiscus & Nacional B \\
\hline 12. Distúrbios de Comunicação & 1 & Lilacs, Rede Saci, & Nacional B \\
\hline 13. Revista Pediátrica Moderna & 1 & Lilacs, Index Internacional De Pediatria & Nacional B \\
\hline 14. Revista da Sociedade de Psicoterapias Analíticas Grupais do & 1 & Lilacs. Index Psi, PePsic & Nacional B \\
\hline 15. Saúde em Debate & 1 & Lilacs. Hisa & Nacional B \\
\hline 16. Revista Diagnóstico \& Tratamento & 1 & Lilacs & Nacional B \\
\hline 17. Acta Ortopédica Brasileira & 4 & Scielo, Lilacs & Nacional A \\
\hline 18. Revista Brasileira de Educação Especial & 4 & Scielo, Edubase, Clase, Psicodoc & Nacional A \\
\hline 19. Paidéia & 2 & Scielo, Lilacs, Psycinfo, Indexpsi, & Nacional A \\
\hline 20. Revista Paulista de Pediatria & 2 & Scielo, Lilacs & Nacional A \\
\hline 21. Revista Produção & 2 & Scielo, REBAE & Nacional A \\
\hline 22. Revista Brasileira de Medicina do Esporte & 1 & Scielo, Lilacs, Embase & Nacional A \\
\hline 23. Revista Brasileira de Ortopedia & 1 & Scielo, Lilacs & Nacional A \\
\hline Total & 162 & & \\
\hline
\end{tabular}


OLIVER, F. C. Pesquisa e produção bibliográfica em terapia. Rev. Ter. Ocup. Univ. São Paulo, v. 19, n. 2, p. 108-120, maio/ago. 2008.

Na Tabela 2, observa-se 110 artigos publicados em revistas nacionais e estrangeiras, 58 deles em periódicos de terapia ocupacional, que não estão inclusos nas bases de dados anteriores, alguns que podem ser acessados pela rede mundial de computadores e ainda outros 30 artigos publicados em revistas sobre as quais não foram localizadas informações, situação semelhante ao estudo anterior. Por outro lado, chama a atenção, também, a presença de cinco artigos em revistas pertencentes a bases de dados da educação, psicologia, saúde e trabalho, sendo dois deles na base Pascal/Francis. A base de dados Pascal (ciências, tecnologia e medicina) e a Francis (ciências humanas e sociais) são européias e organizadas pelo Institut de L'Ínformation Scientifique et Techinique do Centre National de La Recherche Scientifique (CNRS) na França. Essa base congrega documentos em 100 línguas diferentes com predominância do inglês $(63 \%)$ do francês $(12 \%)$, do alemão ( $8 \%$ ) e de outras $(7 \%)$ e é utilizada em 24 países para a produção de indicadores bibliométricos (FARIA et al.2002). Estudo realizado por Mugaini et al. (2004) indicou as bases Pascal e Francis como importantes para a avaliação e divulgação da produção científica brasileira, que poderia ser utilizada para construir indicadores de avaliação, complementarmente, aos dados advindos do ISI.

Tabela 2 - Artigos publicados em periódicos nacionais ou estrangeiros, segundo indexadores, periodicidade e acesso, 2001 a 2007

\begin{tabular}{|c|c|c|c|c|c|}
\hline Periódico & No. & Indexador & $\begin{array}{l}\text { Periodici- } \\
\text { dade }\end{array}$ & Acesso & Sítio eletrônico \\
\hline $\begin{array}{l}\text { Cadernos de Terapia Ocupacio- } \\
\text { nal UFSCar }\end{array}$ & 43 & Não localizado & semestral & impresso & $\begin{array}{l}\text { http://www.ufscar.br/portugues/centros/ccbs/dto/cader- } \\
\text { nos_to.htm }\end{array}$ \\
\hline $\begin{array}{l}\text { Revista do Centro de Estudos de } \\
\text { Terapia Ocupacional }\end{array}$ & 7 & Não localizado & anual & impresso & $\begin{array}{l}\text { http://ceto.terapiaocupacional.googlepages.com/revista- } \\
\text { ceto }\end{array}$ \\
\hline Revista Univap & 7 & Não localizado & semestral & on line & $\begin{array}{l}\text { http://www.univap.br/univap/pro_reitorias/cultura_divul- } \\
\text { gacao }\end{array}$ \\
\hline $\begin{array}{l}\text { Revista de Terapia Ocupacional } \\
\text { da Bahiana }\end{array}$ & 6 & Não localizado & anual & impresso & $\begin{array}{l}\text { http://www.bahiana.edu.br/novosite/hp/revistas_baiana. } \\
\text { asp\# }\end{array}$ \\
\hline $\begin{array}{l}\text { Arquivos Brasileiros de } \\
\text { Paralisia Cerebral }\end{array}$ & 6 & Não localizado & - & - & (1) \\
\hline $\begin{array}{l}\text { Revista de Estudos } \\
\text { Universitários }\end{array}$ & 3 & Não localizado & - & impresso & http://www.uniso.br/publicacoes/revistas/estudos.asp \\
\hline Pesquisa em Saúde & 3 & Não localizado & - & - & - \\
\hline $\begin{array}{l}\text { Revista Chilena de Terapia } \\
\text { Ocupacional }\end{array}$ & 2 & Não localizado & anual & on line & http:// www.terapiaocupacional.cl \\
\hline $\begin{array}{l}\text { Cadernos Centro Universitário } \\
\text { São Camilo }\end{array}$ & 2 & Não localizado & trimestral & impresso & http://www.saocamilo-sp.br/novo/publicacoes/ \\
\hline Prática Hospitalar & 2 & Não localizado & bimensal & impresso & http://www.praticahospitalar.com.br/ \\
\hline Revista Médica de Minas Gerais & 2 & Não localizado & $\begin{array}{l}\text { Não infor- } \\
\text { mada }\end{array}$ & on line & http://www.smp.org.br/atualizacao/revista_disponivel.php \\
\hline $\begin{array}{l}\text { Travailler - Revue Internacio- } \\
\text { nale de Psychopathologie et de } \\
\text { Psychodynamique du Travail }\end{array}$ & 1 & Pascal/Francis & semestral & on line & http:// www.cairn.info/revue-travailler.htm \\
\hline $\begin{array}{l}\text { Cadernos de Psicologia Social } \\
\text { do Trabalho }\end{array}$ & 1 & Index Psi, PePsic & semestral & on line & http:// pepsic.bvs-psi.org.br \\
\hline Pro-Posições & 1 & $\begin{array}{l}\text { Francis,BIBE, } \\
\text { BBE, EDUBA- } \\
\text { SE; Carfax,Iresie }\end{array}$ & trimestral & on line & http:// mail.fae.unicamp.br/ proposicoes/edições/home \\
\hline $\begin{array}{l}\text { Revista Brasileira de Saúde } \\
\text { Ocupacional }\end{array}$ & 1 & $\begin{array}{l}\text { CIS/ILO, Latin- } \\
\text { dex, Repdisca, }\end{array}$ & semestral & on line & http://www.fundacentro.org.br/rbso \\
\hline Revista Histedbr On-Line & 1 & Edubase & trimestral & on line & $\begin{array}{l}\text { http://www.histdbr.fae.unicamp.br/revista/edicoes/30/ } \\
\text { index.htm }\end{array}$ \\
\hline $\begin{array}{l}\text { Revista Chilena de Antropolo- } \\
\text { gia Visual }\end{array}$ & 1 & Não localizado & semestral & on line & http:// www.antropologiavisual.cl \\
\hline Outras* & 21 & - & - & - & - \\
\hline Total & 110 & & & & \\
\hline
\end{tabular}

* são 21 revistas (19 nacionais e 2 estrangeiras) cada uma com 1 artigo publicado, sobre as quais não foram encontradas informações 
OLIVER, F. C. Pesquisa e produção bibliográfica em terapia. Rev. Ter. Ocup. Univ. São Paulo, v. 19, n. 2, p. 108-120, maio/ago. 2008.

Quando se observa, na Tabela 3, a distribuição de artigos em periódicos nacionais e estrangeiros classificados como Internacionais $\mathrm{A}, \mathrm{B}$ ou $\mathrm{C}$ pela Capes, percebe-se a dispersão em 27 periódicos, 17 nacionais (responsáveis pela divulgação de 59 artigos) e 10 estrangeiros (com 17 artigos). Os periódicos nacionais são de áreas como saúde pública, crescimento e desenvolvimento, psicologia, educação, fisioterapia e saúde. Já os periódicos estrangeiros incluem quatro do âmbito da terapia ocupacional (World Federation of Occupational Therapy Bulletin, Occupational
Therapy International, Canadian Journal of Occupational Therapy e Journal of Hand Therapy), três do campo da reabilitação (Disability and Rehabilitation, Journal of Rehabilitation e Manual Therapy) e os demais relacionados a áreas afins.

Um outro aspecto a se discutir é a dispersão de artigos em 88 periódicos (15 estrangeiros e 73 nacionais), o que divulga a área em diferentes campos do conhecimento, mas também dificulta a consolidação dos periódicos de terapia ocupacional nacionais.

Tabela 3 - Artigos publicados em periódicos nacionais e estrangeiros classificados pela Capes como Qualis Internacional, segundo indexadores, 2001 e 2007

\begin{tabular}{|c|c|c|c|}
\hline Periódico & No. Artigos & Indexadores & Qualis Capes \\
\hline 1. Revista Brasileira de Fisioterapia* & 13 & $\begin{array}{l}\text { CSA, Lilacs, Scielo, Latindex, SportDiscus; Science Citation Expanded (Sci- } \\
\text { Search); }\end{array}$ & Internacional $\mathrm{C}$ \\
\hline 2. Arquivos de Neuro-Psiquiatria & 10 & $\begin{array}{l}\text { Medline, Lilacs; Scielo, Embase, Periodica; Neuroscience Citation Index ISI, } \\
(J C R \text { - Impacto 0,441) }\end{array}$ & Internacional B \\
\hline 3. Interface & 10 & Lilacs, Scielo, Latindex, Clase, Encare; Sociological Abstracts & Internacional $\mathrm{C}$ \\
\hline 4. Revista Fisioterapia e Pesquisa & 4 & Lilacs, Latindex, Cinahl, Sport Discus & Internacional C \\
\hline 5. Revista Brasileira de Saúde Materno Infantil & 4 & $\begin{array}{l}\text { Scielo, lilacs Latindex, Global Health, Sociological Abstracts; Linguistic \& } \\
\text { Language }\end{array}$ & Internacional $\mathrm{C}$ \\
\hline 6. Cadernos de Saúde Pública & 3 & Lilacs, Medline, Scielo, Cab Abstracts, Index Medicus & Internacional $\mathrm{C}$ \\
\hline 7. Fisioterapia Em Movimento & 3 & Lilacs, IBICT, Cinahl & Internacional C \\
\hline 8. Revista Latino-Americana de Enfermagem* & 2 & Lilacs, Medline, Scielo, Cinahl Psycinfo, Latindex & Internacional C \\
\hline $\begin{array}{l}\text { 9. Revista Brasileira de Crescimento e } \\
\text { Desenvolvimento Humano }\end{array}$ & 2 & Lilacs, Adsaúde, Cinahl & Internacional $\mathrm{C}$ \\
\hline 10. Saúde e Sociedade* & 1 & Scielo, Sociological Abstracts & Internacional $\mathrm{C}$ \\
\hline 11. Estudos de Psicologia & 1 & Scielo, Lilacs, PsycInfo, Psicodoc, IndexPsi Sociological Abstracts & Internacional $\mathrm{C}$ \\
\hline 12. Psicologia em Estudo & 1 & Sociological Abstracts & Internacional $\mathrm{C}$ \\
\hline 13. Revista de Saúde Pública & 1 & $\begin{array}{l}\text { Lilacs, Medline, Scielo, Embase, Excerpta Medica. Journal Citation Reports } \\
\text { (JCR Impacto 0,557) }\end{array}$ & Internacional B \\
\hline 14. Revista de Medicina (Fmrp - USP) & 1 & Medline; Lilacs, Embase & Internacional $\mathrm{C}$ \\
\hline 15. Fisioterapia Brasil & 1 & Lilacs, Cinahl, Latindex & Internacional $\mathrm{C}$ \\
\hline 16. História,Ciências,Saúde & 1 & Lilacs, Medline, Scielo, Latindex;Clase & Internacional $\mathrm{C}$ \\
\hline 17 Revista de Ciências Humanas & 1 & Sociological Abstracts & Internacional C \\
\hline 18. Disability and Rehabilitation & 5 & $\begin{array}{l}\text { Medline, Psycinfo, Embase } \\
\text { (JCR Impacto 1,164) }\end{array}$ & Internacional A \\
\hline 19. Developmental Med And Child Neurology & 2 & $\begin{array}{l}\text { Embase Medline } \\
\text { (JCR Impacto 2,008) }\end{array}$ & Internacional A \\
\hline 20. Gait \& Posture & 1 & Medline, Embase, (JCR Impacto 1,976) & Internacional A \\
\hline 21. Neuroscience Letters & 1 & Embase, Medline (JCR Impacto 2,092) & Internacional A \\
\hline 22. Manual Therapy & 1 & $\begin{array}{l}\text { Medline, EMBASE; CINAHL, } \\
\text { (JCR Impacto 1,931) }\end{array}$ & Internacional A \\
\hline 23. Journal of Rehabilitation & 1 & Medline, Embase (JCR Impacto 0,729) & Internacional A \\
\hline 24. Journal of Hand Therapy & 1 & Medline, Embase, Cinahl & Internacional $\mathrm{C}$ \\
\hline 25. Occupational Therapy International & 1 & Medline, Cinahl, Psycinfo, Embase & Internacional $\mathrm{C}$ \\
\hline $\begin{array}{l}\text { 26. World Federation of Occupaciional Therapy } \\
\text { bulletin }\end{array}$ & 3 & Cinahl & Internacional $\mathrm{C}$ \\
\hline 27. Canadian Journal of Occupational Therapy & 1 & Medline, Cinahl, Psycinfo, Embase & Internacional C \\
\hline Total & 76 & & \\
\hline
\end{tabular}


OLIVER, F. C. Pesquisa e produção bibliográfica em terapia. Rev. Ter. Ocup. Univ. São Paulo, v. 19, n. 2, p. 108-120, maio/ago. 2008.

Vale ressaltar também a importante participação dos pesquisadores na construção dos periódicos de terapia ocupacional, conforme apresentado na Tabela 4 . No período do estudo, os pesquisadores foram responsáveis por cerca de $70 \%$ dos artigos publicados na Revista de Terapia Ocupacional da USP, cerca de 50\% dos publicados nos Cadernos de Terapia Ocupacional da UFSCar e 26,1\% dos artigos da Revista de Terapia Ocupacional da Bahia. Os periódicos da área têm sido os veículos de divulgação da produção científica de pesquisadores, o que indica a sua importância para a constituição e consolidação da terapia ocupacional no país. É fundamental a continuidade da participação de pesquisadores nesses veículos como também de profissionais brasileiros e assim possibilitar a inclusão da terapia ocupacional em bases de dados de acesso livre, como é o caso do ScieLO, que tem grande abrangência nacional e na América Latina. Participar de bases de dados dessa natureza contribui para a divulgação da área e para tornar públicas as atividades de pesquisa realizadas nesse campo de conhecimento utilizando-se de importante mecanismo de comunicação e divulgação científica como é a rede mundial de computadores (CASTRO, 2006).

Tabela 4 - Participação de artigos de pesquisadores publicados nos periódicos de terapia ocupacional no período de 2001 a 2007

\begin{tabular}{|c|c|c|c|}
\hline Periódicos & No. de artigos do periódico & No. de artigos de pesquisadores & Freq $(\%)$ \\
\hline Revista de Terapia Ocupacional da USP* & 130 & 97 & 74,6 \\
\hline Cadernos de Terapia Ocupacional da UFSCar & 76 & 43 & 56,5 \\
\hline Revista de Terapia Ocupacional do CETO** & 36 & 7 & 19,5 \\
\hline Revista de Terapia Ocupacional da Bahiana*** & 23 & 6 & 26,1 \\
\hline Total & 265 & 153 & 100.0 \\
\hline
\end{tabular}

\section{Desafios para o desenvolvimento da pesquisa em Terapia Ocupacional: algumas considerações}

O perfil de produção bibliográfica da área está em constituição, mesclando a produção intelectual sob forma de livros e de capítulos de livros, com a produção de artigos, de forma crescente, no período do estudo. Há um esforço concreto dos pesquisadores em lidar com as demandas de um campo de conhecimento, que implica discussão de saberes e de práticas, tanto no âmbito da pesquisa como da formação profissional.

É importante que tanto pesquisadores como estudantes de graduação e pós-graduação de terapia ocupacional reconheçam o percurso trilhado nos últimos anos e percebam seus limites e a necessidade de estabelecimento de estratégias de curto, médio e longo prazos para o desenvolvimento da pesquisa e da divulgação científica em terapia ocupacional.

E nesse sentido é importante refletir sobre a repercussão do atual sistema de avaliação da produção intelectual e de pesquisa, que tem privilegiado, a divulgação de artigos em periódicos indexados em determinadas bases de dados internacionais, que trazem limites objetivos ao desenvolvimento da pesquisa no país como apontam Coimbra Jr (1999) e Castiel e Sanz-Valero (2007).

Essa prática institucional, que é critério para criação e avaliação da qualidade de programas de pós-graduação, bem como para a distribuição de recursos financeiros, como indicaram Castiel e Sanz-Valero (2007) torna-se um importante fator de organização de relações institucionais e políticas no âmbito das comunidades científicas no país. Para esses autores, o produtivismo acadêmico transformou o artigo científico em uma mercadoria acadêmica, que modifica relações institucionais e de desenvolvimento de pesquisa com amplas repercussões na formação e no delineamento de pesquisas. Essa dinâmica tem possibilitado transformar o trabalho intelectual, como apontaram Rocha e Rocha (2004) e Luz (2005), aos moldes do trabalho industrial, a partir da lógica da produtividade/mercadoria e da competitividade, processos vivenciados pelos trabalhadores/pesquisadores/ intelectuais, também submetidos a tempos e padrões de produtividade, na maior parte das vezes, exteriores ao processo de reflexão e de desenvolvimento de pesquisa, principalmente aquela do campo das ciências humanas. A máxima "publicar ou morrer" (EVANGELISTA, 2002) tem transformado as perspectivas de pesquisa e de capacitação 
de pesquisadores do país, em todas as áreas do conhecimento, mas com especial influência sobre aquelas em processo de constituição, como é o caso da terapia ocupacional.

A ênfase do sistema de avaliação na divulgação de artigos dificulta o investimento em outros tipos de produção intelectual, como aqueles representados pelos livros ou capítulos de livros, que não têm relevância nas bases de dados internacionais como o ISI, e que como afirmam Carvalho e Manoel (2006, p. 211) também "torna questionável a postura desse instituto, no que diz respeito a mapear a produção científica mundial".

Nesse sentido, há esforços no interior da comunidade acadêmica, para discutir e apresentar um sistema de classificação Qualis Livro, a partir da experiências brasileiras das diferentes áreas do conhecimento, mas que ainda ocupa posição de menor valor diante da produção de artigos (CARVALHO; MANOEL, 2006).

$\mathrm{O}$ privilégio à produtividade além de parcelar as atividades de pesquisa produzindo estudos orientados pelo produtivismo, induz a maior parte dos pesquisadores a publicar em periódicos internacionais em detrimento dos nacionais, local privilegiado para o diálogo acadêmico e para o retorno de resultados dos investimentos nacionais em pesquisa.

Vale a pena refletir sobre qual é o sentido e a necessidade concreta de divulgação de trabalhos fora do país, para quais áreas e sobre quais temas? Em que medida essa pode ser uma preocupação e necessidade para os pesquisadores em terapia ocupacional? Victora e Moreira (2006), em estudo sobre autores brasileiros em saúde coletiva, discutem as dificuldades encontradas para divulgação de resultados de pesquisas da área da saúde coletiva em periódicos dos países do hemisfério Norte (de alta renda). Nesse estudo, uma das dificuldades referidas pelo autores da área de saúde coletiva era a existência de preconceito editorial de revistas internacionais para a publicação de trabalhos acadêmicos de países do hemisfério Sul (de renda média ou baixa) assim como as questões relativas ao idioma e prioridades editoriais.

No caso da terapia ocupacional, as dificuldades para divulgação e desenvolvimento de pesquisa são diversas. Há questões estruturais, como o pequeno número de doutores no país, poucos deles vinculados a atividades de pós- graduação. Além disso, a inexistência de pós-graduação em terapia ocupacional é resultado, em parte, desse sistema de classificação da produção intelectual, que tem privilegiado a análise da produção bibliográfica sobre determinados parâmetros de produtividade, em detrimento das necessidades de capacitação docente e para a pesquisa, como ainda é o caso da terapia ocupacional. A expansão recente dos cursos de graduação, inclusive nas universidades públicas e em diferentes regiões do país, traz a necessidade de se estruturar processos de capacitação docente no âmbito de estudos de mestrado e doutorado, que poderiam ser realizados, também, a partir de programas específicos na área. Esse é um tema que deve ser levado às Universidades e à CAPES, pois nem todas as áreas do conhecimento já constituíram seu corpo de pesquisadores doutores.

Em se mantendo esse sistema que associa numa mesma agência processos de avaliação e de destinação de recursos para pesquisa (sob a forma de bolsas, principalmente) torna-se difícil para áreas em constituição e emergentes discutirem e disputarem critérios de avaliação, que, em última estância, estão restringindo o acesso aos recursos financeiros para pesquisa, ainda escassos no Brasil.

As dificuldades encontradas para o desenvolvimento da pós-graduação em terapia ocupacional trazem repercussões diretas para a formação profissional na graduação e para o reconhecimento da importância da participação da área nas atividades assistenciais.

Por outro lado, a vinculação da pesquisa e pósgraduação apenas à grande área da saúde dificulta o desenvolvimento de pesquisas na interface entre diferentes campos de conhecimento, que tem constituído, também, a singularidade da terapia ocupacional brasileira.

No entanto, os desafios apresentados não devem impedir o reconhecimento dos esforços e dos resultados obtidos pelos pesquisadores de terapia ocupacional participantes deste estudo. Reconhecer os percursos trilhados é essencial para a continuidade dos investimentos pessoais e institucionais no âmbito da construção de pesquisa e da divulgação científica em terapia ocupacional e possibilita a continuidade de pesquisas sobre o tema, qualificando forma o debate acadêmico e institucional necessário ao desenvolvimento do campo da terapia ocupacional no país.

Agradecimentos: À terapeuta ocupacional Renata Beltrame pela preparação do banco de dados e à Profa. Dra. Eucenir Fredini Rocha pelo apoio na definição dos principais aspectos a serem trabalhados neste texto. 
OLIVER, F. C. Pesquisa e produção bibliográfica em terapia. Rev. Ter. Ocup. Univ. São Paulo, v. 19, n. 2, p. 108-120, maio/ago. 2008.

OLIVER, F. C. Research and bibliographic production on occupational therapy: contributions to the debate on parameters for the evaluation of brazilian academic production. Rev. Ter. Ocup. Univ. São Paulo, v. 19, n. 2, p. 108-120, maio/ago. 2008.

\begin{abstract}
This study characterized the bibliographic production (2001 to 2007) of research groups, coordinated by occupational therapists, included in CNPq Directory of Research Groups. Seventeen research groups were identified, 12 of which in public institutions. There was an increase (68\%) of participants in the groups (2000 to 2007). The groups sum $48 \mathrm{PhDs}, 12$ of which are linked to graduate programs stricto sensu. The researchers published 21 books, 159 book chapters and 348 papers. It is noticeable the predominance of articles published in national journals; 146 $(41,7 \%)$ of those were published in journals classified as National B, $16(4,6 \%)$ as National A. The articles published in journals classified as international circulation represent $21,7 \%$, with $54(15,5$ $\%)$ International C, $11(3,1 \%)$ International B and 11(3,1\%) International A. The groups' researchers were responsible for $153(57,7 \%)$ of the 265 papers published in the occupational therapy journals, an important fact for the maintenance of these periodicals. Papers are visibly spread throughout a great number of national journals, which divulge the area, but it makes it harder for the consolidation of occupational therapy journals. The positive changes support the evaluation of the contributions of Brazilian authors to the area; and better qualify the bibliographical production and research in occupational therapy.
\end{abstract}

KEY WORDS: Occupational therapy. Research. Bibliography. Research groups. Scientific publication indicators.

\title{
REFERÊNCIAS
}

BARROS, D. D.; OLIVER, F. C. Contribuição para a discussão do Qualis de terapia ocupacional no Brasil. Rev. Ter. Ocup. Univ. São Paulo, v. 14, n,. 2, p. 52-63, , 2003.

CARVALHO, Y. M.; MANOEL, E. de J. Para além dos indicadores de avaliação da produção intelectual na grande área da saúde Movimento, Porto Alegre, v. 12, n. 3, p. 193-225, 2006.

CASTIEL, L. D.; SANZ-VALERO, J. Entre fetichismo e sobrevivência: o artigo científico é uma mercadoria acadêmica?. Cad. Saúde Pública, Rio de Janeiro, v. 23, n. 12, 2007. Disponível em: http://www.scielo.br/scielo.php?script=sci_arttext\&pid=S0102$311 \mathrm{X} 2007001200026 \& \operatorname{lng}=$ pt\&nrm=iso. Acesso em: 28 jul. 2008. doi: 10.1590/S0102-311X2007001200026.

CASTRO, R. C. F. Impacto da Internet no fluxo da comunicação científica em saúde. Rev. Saúde Pública, São Paulo, v. 40, n. esp., ago. 2006 . Disponível em: http://www.scielo.br/ scielo.php?script=sci_arttext\&pid=S0034-89102006000400009$\& \operatorname{lng}=$ pt\&nrm=iso. Acesso em: 18 ago. 2008. doi: $10.1590 /$ S003489102006000400009.

COIMBRA JR., C. E. A.. Produção científica em saúde pública e as bases bibliográficas internacionais. Cad. Saúde Pública, Rio de Janeiro, v. 15, n. 4, 1999 . Disponível em: http://www. scielosp.org/scielo.php?script $=$ sci_arttext\&pid=S0102-311$\mathrm{X} 1999000400022 \& \operatorname{lng}=$ pt\&nrm=iso. Acesso em: 20 abr. 2008. doi: 10.1590/S0102-311X1999000400022.

CONSELHO FEDERAL DE FISIOTERAPIA E TERAPIA OCU-
PACIONAL - COFFITO. Distribuição de terapeutas ocupacionais no país em setembro de 2008. (comunicação pessoal)

COORDENAÇÃO DE APERFEIÇOAMENTO DE PESSOAL DE NÍVEL SUPERIOR - Capes Critério para Classificação de Periódicos no Sistema Qualis: grande área de Saúde triênio 2004 -2006. Disponível em: http:/qualis.capes.gov.br/arquivos/avaliacao/webqualis/criterios2004_2006/Criterios_Qualis_2005_21.pdf Acesso em: 20 abr. 2008.

EMMEL, M. L. G. Caracterização dos cursos de terapia ocupacional do Brasil: perspectivas frente à capacitação docente. Relatório de pesquisa. São Carlos, 2004 (mimeo)

EMMEL, M. L. G.; LANCMAN, S. Quem são nossos mestres e doutores/ $\mathrm{O}$ avanço da capacitação docente em terapia ocupacional no Brasil. Cad. Ter. Ocup. UFSCar, São Carlos, v. 7, n. 1, p. 29-38, 1998a.

EMMEL, M. L. G.; LANCMAN, S. A influência da capacitação dos terapeutas ocupacionais no processo de constituição da profissão no Brasil. Cad. Ter. Ocup. UFSCar, São Carlos, v. 7, n. 2, p. 49-57, 1998 b.

EVANGELISTA, O.. Publicar ou morrer In: BIANCHETTI, L.; MACHADO NETTO, A. M. (orgs.). A bússola do escrever: desafios e estratégias na orientação de teses e dissertações. Florianópolis: Ed. UFSC; São Paulo: Cortez, 2002. p. 297-301.

FARIA, L. I. L.; QUONIAM, L.; MUGNAINI, R. Elementos de 
comparação das bases de dados Pascal e SCISEARCH. Int. J. Inf. Scie. Decision Making, Toulon - Var, v. 5 p. 30, 2002

INSTITUTO DE PESQUISAS EDUCACIONAIS ANÍSIO TEIXEIRA - INEP. Cursos de graduação. Disponível em: http://www. educacaosuperior.inep.gov.br/funcional/busca_curso.stm Acesso em: 30 jul 2008.

LUZ, M. T. Prometeu acorrentado: análise sociológica da categoria produtividade e as condições atuais da vida acadêmica. Physis, Rio de Janeiro, v. 15, n. 1, jun. 2005. Disponível em: http://www.scielo.br/scielo.php?script=sci_arttext\&pid=S0103$73312005000100003 \& \operatorname{lng}=$ pt\&nrm=iso. Acesso em: 20 ago 2008. doi: 10.1590/S0103-73312005000100003.

MAGALHÃES, L. Situação da capacitação docente e perspectivas de criação de cursos de pós-graduação em terapia ocupacional na Universidade Federal de Minas Gerais - UFMG. Rev. Ter. Ocup. Univ. São Paulo, v. 9, n. 3, p. 119-24, 1998.

MUGNAINI, R.; JANNUZZI, P. de M.; QUONIAM, L. Indicadores bibliométricos da produção científica brasileira: uma análise a partir da base Pascal. Ci. Inf. , Brasília,v. 33, n. 2, ago. 2004. Disponível em: http://www.scielo.br/scielo. php?script $=$ sci_arttext\&pid $=$ S0100-19652004000200013$\& \operatorname{lng}=$ pt\&nrm=iso. Acesso em: 15 mar. 2008. doi: 10.1590/ S0100-19652004000200013.

ROCHA, M. L. da; ROCHA, D. Produção de conhecimento, práticas mercantilistas e novos modos de subjetivação. Psicol. Soc., Porto Alegre, v. 16, n. 1, 2004. Disponível em: http:// www.scielo.br/scielo.php?script $=$ sci_arttext\&pid $=$ S0 102 $71822004000100003 \& \operatorname{lng}=$ pt\&nrm=iso. Acesso em: 15 set. 2008. doi: 10.1590/S0102-71822004000100003

TIRADO, M. G.; ALMEIDA, M. C. de; LOPES, R. E.; GALHEIGO, S. Maria. Terapia Ocupacional. In: HADDAD, A. E. et al. (org) A trajetória dos cursos de graduação na área da saúde: 1991 - 2004. Brasília: Instituto de Pesquisas Educacionais Anísio Teixeira, 2006. p. 399-528.

VICTORA, C. G; MOREIRA, C. B. Publicações científicas e as relações Norte-Sul: racismo editorial?. Rev. Saúde Pública . São Paulo, v. 40, n. esp, ago. 2006. Disponível em: http://www.scielo.br/ scielo.php?script=sci_arttext\&pid=S0034-89102006000400006$\& \operatorname{lng}=$ pt\&nrm=iso. Acesso em: 20 abr. 2008. doi: 10.1590/S003489102006000400006. 